Steven Bank

Nagoya Math. J.

Vol. 39 (1970), 107-117

\title{
ON THE GROWTH OF SOLUTIONS OF ALGEBRAIC DIFFERENTIAL EQUATIONS WHOSE COEFFICIENTS ARE ARBITRARY ENTIRE FUNCTIONS ${ }^{1}$
}

\author{
STEVEN BANK
}

1. Introduction: In this paper we treat the problem of determining the rate of growth of entire functions which are solutions of first order algebraic differential equations whose coefficients are arbitrary entire functions (i.e. equations of the form $\Omega(z, y, d y / d z)=0$, where $\Omega(z, y, d y / d z)=$ $\sum_{k, j \geq 0} f_{k j}(z) y^{k}(d y / d z)^{j}$ is a polynomial in $y$ and $d y / d z$, whose coefficients $f_{k \jmath}(z)$ are entire functions).

In [7], Valiron treated the special case where the coefficients $f_{k j}(z)$ are polynomials, and in this case, it was shown ([7; p. 41]) that any entire solution must be of finite order. In [1], the author considered the special case where the coefficients $f_{k j}(z)$ are entire functions of finite order. Clearly such equations can possess entire solutions of infinite order (for example, $\exp (\exp z), \sin (\cos z))$, but it was shown in [1] that when all the coefficients are of finite order, the growth of an entire solution $h(z)$ is restricted in the following natural way: For any real number $\lambda$ which is greater than the order of each coefficient $f_{k j}(z)$, the maximum modulus $M(r ; h)$ of $h(z)$ satisfies the inequality $M(r ; h) \leq \exp \left(\exp r^{\lambda}\right)$ for all $r$ greater than some number $r_{0}(\lambda)$.

In this paper, we treat the general case where the coefficients $f_{k j}(z)$ are arbitrary entire functions (i.e. each coefficient can be of finite or infinite order). This is accomplished in part through the use of the concept of "an order" of an entire function $f(z)$, which was introduced by Blumenthal in [2] in the case when $f(z)$ is of infinite order. Roughly speaking, an order of $f(z)$ is a special kind of monotone nondecreasing function $\mu(r)$ with the property that $M(r ; f) \leq \exp \left(r^{\mu(r)}\right)$ for all sufficiently large $r$. (For the

Received March 17, 1969.

1) This research was supported in part by the National Science Foundation (GP 7374). 
reader's convenience, the definition of "an order" as introduced in [2], is reviewed in $\S 2$ below). Our main result here ( $\$ 3$ below) states the following: Let $\varphi(r)$ be a monotone nondecreasing function greater than 1 , which for all sufficiently large $r$, is greater than the order of each coefficient having finite order, and is greater than an order of each coefficient having infinite order. Then for any entire solution $h(z)$ of $\Omega(z, y, d y / d z)=0$ and any real number $a>1$, the inequality $M(r ; h) \leq \exp \left(\exp r^{\varphi(a r)^{a}}\right)$ holds for all $r$ greater than some number $r_{0}(a)$.

In $\S 5$, we consider the application of our main result to meromorphic solutions of $\Omega(z, y, d y / d z)=0$.

The principal tools used in the proof of the main result consist of (i) the Wiman-Valiron theory of the maximum term $([9,10,11]$ or for a complete discussion [6; Chapter 9]); (ii) Certain results ([2; p. 97] and [5; p. 336]) concerning the minimum modulus of entire functions, and (iii) Certain results ([9; pp. 239-240]), concerning the relation between the maximum modulus and maximum term of an entire function.

2. We review here briefly the concept of order for an entire function $f(z)$. Let $M(r ; f)=\max _{|z|=r}|f(z)|$ and let $\nu(r)$ be defined by the relation $M(r ; f)=\exp \left(r^{\nu}(r)\right) . \quad$ The order of $f$ is $\lambda=\limsup _{r \rightarrow+\infty} \nu(r) . \quad$ If $\lambda=+\infty$, then an order of $f$, as defined in [2; pp. 21, 22,31], is a monotone nondecreasing function $\mu(r)$, defined on an interval $[A,+\infty)$, satisfying the following three conditions :

(I) There exists a continuous decreasing function $\varepsilon(r)$ on $[A,+\infty)$, with $\lim _{r \rightarrow+\infty} \varepsilon(r)=0$, such that for each $r \in[A,+\infty), \mu\left(r_{1}\right) \leq \mu(r)^{1+\varepsilon(r)}$, where $r_{1}=r^{1+(1 / \mu(r)) \varepsilon(r)}$.

(II) For all $r \in[A,+\infty), \nu(r) \leq \mu(r) \quad\left(\right.$ so $\left.M(r ; f) \leq \exp \left(r^{\mu(r)}\right)\right)$.

(III) There exists a continuous decreasing function $\delta(r)$ on $[A,+\infty)$, with $\lim _{r \rightarrow+\infty} \delta(r)=0$, such that for a sequence or values of $r$ tending to $+\infty$, $\mu(r)<\nu(r)^{1+\delta(r)}$.

It is proved in [2; pp. 23-24] that orders exist for every entire function of infinite order. Also developed in [2], is a method for estimating the magnitude of an order, which roughly states the following: If $\mu_{i}(r)$ is a monotone nondecreasing function satisfying (I) and (II) above for a certain type of $\varepsilon(r)$, then there is an order $\mu(r)$ of $f$, such that $\mu(r) \leq \mu_{1}(r)$ for all 
$r \in[A,+\infty)$. For a complete discussion, the reader is referred to $[2 ; \mathrm{pp}$. 117-128].

3. We now state our main result:

Theorem. Let $\Omega(z, y, d y / d z)=\sum_{k, j \geq 0} f_{k j}(z) y^{k}(d y / d z)^{\jmath}$ be a polynomial in $y$ and $d y / d z$, where each coefficient $f_{k j}(z)$ is an entire function. For each coefficient $f_{k j}(z)$ having infinite order, let $\mu_{k j}(r)$ be an order of $f_{k j}(z)$. If $f_{k j}(z)$ has finite order $\lambda_{k j}$, set $\mu_{k j}(r)$ identically equal to $\lambda_{k j}$. Let $\varphi(r)$ be a monotone nondecreasing function on an interval $\left[R_{0},+\infty\right)$, such that for all $r \in\left[R_{0},+\infty\right), \varphi(r)>1$ and $\mu_{k j}(r)<\varphi(r)$ for each $(k, j)$. Let $h(z)$ be an entire function which satisfies $\Omega\left(z, h(z), h^{\prime}(z)\right) \equiv 0$, and let $M(r ; h)=\max _{|z|=r}|h(z)|$. Then for any real number $a>1$, there exists a positive real number $r_{0}(a)$ such that $M(r ; h) \leq \exp \left(\exp r^{\varphi(a r)^{a}}\right)$ for all $r>r_{0}(a)$.

4. Proof of the Theorem: If $h(z)$ is a polynomial, clearly the result holds. Hence we may assume that

(1) $h$ is an entire transcendental function. Let $\sum_{n=0}^{\infty} c_{n} z^{n}$ be the power series expansion of $h(z)$, and let $M(r)=\max _{|z|=r}|h(z)|$. For each $r \geq 0$, let $N(r)$ be the central index [3; p. 183] of $h$ (i.e. $N(r)$ is the maximum $j$ such that $\left.\left|c_{j}\right| r^{j}=\max _{m \geq 0}\left|c_{m}\right| r^{m}\right)$. Then in view of (1),

(2) $N(r)$ is an unbounded monotone nondecreasing function of $r$, and it is proved in [6; pp. 198, 210] (and also in [8; pp. 95, 103]) that there exists $\alpha \in(0,1)$ such that if we exclude from the interval $(1,+\infty)$ an infinite sequence of exceptional finite open intervals $\left(W_{s}, W_{s}^{\prime}\right)$ for which,

(3) $\sum_{s=1}^{\infty}\left(\log W_{s}^{\prime}-\log W_{s}\right)$ converges, and for which we may assume,

(4) $W_{s}^{\prime}<W_{s+1}$ for all $s$ and $\lim _{s \rightarrow \infty} W_{s}^{\prime}=+\infty$, then in the remaining set $(1,+\infty)-E$, where $E=\bigcup_{s=1}^{\infty}\left(W_{s}, W_{s}^{\prime}\right)$, the following are true: There exists a number $R_{1} \geq 1$ such that for $r>R_{1}$ and $r \notin E$, we have

(5) $\log M(r)>c(N(r))^{\alpha}$, where $c$ is a positive constant independent of $r$, and if $z$ is any point on $|z|=r$ at which $|h(z)|=M(r)$, then

(6) $h^{\prime}(z)=(N(r) / z)(1+\delta(z)) h(z)$, where $|\delta(z)|<N(r)^{-\eta}$ for some fixed $\eta>0$. (These elements of $(1,+\infty)$ are called ordinary values of index $\alpha$ in $[6,7,8])$. 
Now by our assumption concerning $\varphi(r)$, we clearly have that there exists $R_{2}>1$ such that if $r \geq R_{2}$ then

(7) $\left|f_{k j}(z)\right| \leq \exp \left(r^{\varphi(r)}\right)$ on $|z|=r$ for all $(k, j)$.

Now (as in the statement of the theorem) let a be any real number greater than 1 , and set

(8) $\varepsilon=(a-1) / 3$ and $b=\sqrt{a}$. (Thus $\varepsilon>0$ and $b>1)$. Let

(9) $p=\max \left\{k+j: f_{k j} \not \equiv 0\right\}$ and $m=\max \left\{j: f_{p-j, j} \not \equiv 0\right\}$, and consider the coefficient $f_{p-m, m}(z)$. Let $\mu(r)$ be the function $\mu_{p-m, m}(r)$ as in the statement of the theorem.

Suppose first that $f_{p-m, m}(z)$ is of infinite order, so $\mu(r)$ is an order of $f_{p-m, m}$. In this case it is proved in [2; p. 97], that if $d_{1}, d_{2}, \ldots$ is the sequence of zeros of $f_{p-m, m}$, then we can remove from the plane, open disks $\left|z-d_{n}\right|<\theta_{n}(n=1,2, \cdots)$ such that

(10) $\sum_{n \geq 1} \theta_{n}$ converges, and such that in the remaining domain we have

(11) $\left|f_{p-m, m}(z)\right|>\exp \left(-r^{u(r)^{1+\varepsilon}}\right)$ on $|z|=r$, when $r$ is greater than some number $R_{3}^{\prime}$. Letting $F$ be the union of all the open intervals $\left(\left|d_{n}\right|-\theta_{n}\right.$, $\left.\left|d_{n}\right|+\theta_{n}\right)$ for $n=1,2, \cdots$, clearly (11) holds on $|z|=r$ if $r>R_{3}^{\prime}$ and $r \notin F$. By our assumption, $\mu(r)<\varphi(r)$ for $r \geq R_{0}$, so if $R_{3}=\max \left\{R_{0}, R_{3}^{\prime}\right\}$ then by (11),

(12) $\left|f_{p-m, m}(z)\right|>\exp \left(-r^{\varphi(r)^{1+\varepsilon}}\right)$ on $|z|=r$, if $r>R_{3}$ and $r \notin F$. In view of (10), clearly $F$ can be written as the union of a sequence of finite open intervals $\left(T_{s}, T_{s}^{\prime}\right)$ such that

(13) $T_{s}^{\prime}<T_{s+1}$ for all $s$, and $\sum_{s \geq 1}\left(T_{s}^{\prime}-T_{s}\right)$ converges.

We now show that (12) and (13) also hold in the case when $f_{p-m, m}(z)$ is of finite order. In this case $\mu(r) \equiv \lambda$ where $\lambda$ is the order of $f_{p-m, m}(z)$. Now $\varphi\left(R_{0}\right)>1$ by assumption, so $\varepsilon^{\prime}=(1 / 2) \varphi\left(R_{0}\right)\left(\varphi\left(R_{0}\right)^{\varepsilon}-1\right)$ is strictly positive. Let $d_{1}, d_{2}, \cdots$ be the non-zero roots of $f_{p-m, m}$ and let $D$ be the domain obtained by removing from the plane all the disks $\left|z-d_{n}\right|<\left|d_{n}\right|^{-\left(\lambda+\epsilon^{\prime}\right)}$. Then since $\lambda$ is the order of $f_{p-m, m}$ and $\boldsymbol{\varepsilon}^{\prime}>0$, it is proved in [5; p. 328] that

(14) $\sum_{n \geq 1}\left|d_{n}\right|^{-\left(\lambda+\varepsilon^{\prime}\right)}$ converges, and it is proved in [5; p. 336] that there exists $R_{3}^{\prime}>1$ such that for $z \in D$,

(15) $\left.\left|f_{p-m, m}(z)\right| \geq \exp \left(-r^{\lambda+\varepsilon^{\prime}}\right)\right)$ when $|z|=r>R_{3}^{\prime}$. Let $F$ be the union 
of all the open intervals $\left.\left(\left|d_{n}\right|-\left|d_{n}\right|^{-\left(\lambda+\varepsilon^{\prime}\right)}\right),\left|d_{n}\right|+\left|d_{n}\right|^{-\left(\lambda+\varepsilon^{\prime}\right)}\right)$ for $n=1,2, \cdots$. Then it is clear that (15) holds on $|z|=r$ if $r>R_{3}^{\prime}$ and $r \notin F$, and it follows from (14). that $F$ can be written as the union of open intervals $\left(T_{s}, T_{s}^{\prime}\right)$ satisfying (13). As before, let $R_{3}=\max \left\{R_{0}, R_{3}^{\prime}\right\}$. Then for $r>R_{3}, \varphi(r)>1$ and $\lambda=\mu(r)<\varphi(r)$. Thus by definition of $\varepsilon^{\prime}$ and the fact that $\varphi$ is monotone nondecreasing we have $\lambda+\varepsilon^{\prime}<\varphi(r)^{1+\varepsilon}$ for all $r>R_{3}$. In view of (15), we thus obtain (12) even in the case where $f_{p-m, m}(z)$ is of finite order, and so (12) and (13) hold in any case.

In view of (3), (4) and (13), clearly we may write $E \cup F$ as the union of a sequence of finite open intervals,

(16) $\quad E \cup F=\bigcup_{s=1}^{\infty}\left(U_{s}, U_{s}^{\prime}\right)$, where

(17) $U_{s}^{\prime}<U_{s+1}$ for all $s$, and $\lim _{s \rightarrow \infty} U_{s}^{\prime}=+\infty$, and

(18) $\sum_{s=1}^{\infty}\left(\log U_{s}^{\prime}-\log U_{s}\right)$ converges. Now define,

(19) $A=\left\{r \mid r>1\right.$ and $\left.N(r)>\exp \left(3 r^{\varphi(r)^{1+\varepsilon}}\right)\right\}$. We now prove,

Lemma A. There exists a real number $r_{1}>1$ such that $A \cap\left(r_{1},+\infty\right) \subset E \cup F$.

Proof. Assume the contrary. Then there exists a sequence of distinct values of $r$ in $(1,+\infty)$ tending to $+\infty$, such that

(20). $r \in A$ but $r \notin E \cup F$.

Let $B$ be the set of values of $r$ comprising this sequence.

Now $h(z)$ satisfies the relation,

(21) $\sum f_{k j}(h(z))^{k}\left(h^{\prime}(z)\right)^{j} \equiv 0$.

Let $r \in B$ and let $z$ be a point on $|z|=r$ at which $|h(z)|=M(r)$. Then $h(z) \neq 0$ and so by dividing equation $(21)$ by $(h(z))^{p}$ (where $p$ is as in (9)), we can write (21) in the form,

(22) $\sum_{j=0}^{m} f_{p-j, j}(z)\left(h^{\prime}(z) / h(z)\right)^{j}=-\sum_{k+j<p} f_{k_{j}}(z)\left(h^{\prime}(z) / h(z)\right)^{j}(h(z))^{k_{+j}-p}$.

We will denote the left side of $(22)$ by $\Lambda(z)$ and the right side by $\Phi(z)$. Let $R_{4}=\max _{0 \leq j \leq 3} R_{j}$.

We now assert that there exists a real number $R_{5}>R_{4}$ such that if $r \in B$ and $r>R_{5}$ then 
(23) $|\Phi(z)| \leq(M(r))^{-1 / 2} \exp \left(r^{\varphi(r)}\right)$,

at each point of $|z|=r$ at which $|h(z)|=M(r)$.

To prove (23), we recall first from (20) that if $r \in B$ then $r \notin E$ and $r \notin F$. Since $N(r) \rightarrow+\infty$ as $r \rightarrow+\infty$ (by (2)), clearly $\delta(z)$ (in (6)) tends to zero as $r \rightarrow+\infty$ in $B$. Since $M(r)$ also tends to $+\infty$, there exists $R_{4}^{\prime}>R_{4}$ such that for $r \in B$ and $r>R_{4}^{\prime}$, we have

(24) $M(r)>1, N(r)>1$ and $|\delta(z)|<1 / 2$.

Let $r$ be any element of $B$ such that $r>R_{4}^{\prime}$ and let $z$ be any point on $|z|=r$ at which $|h(z)|=M(r)$. We refer to the right side of (22). If $k+j<p$, then $p-(k+j) \geq 1$ so $|h(z)|^{k_{+}-p}=M(r)^{k_{+j}-p} \leq M(r)^{-1}$ (since $\left.M(r)>1\right)$. Since $|\delta(z)|<1 / 2$, we have by (6) that $\left|h^{\prime}(z) / h(z)\right| \leq 2 N(r) / r<2 N(r)$ since $r>1$. Thus $\left.\left|h^{\prime}(z)\right| h(z)\right|^{j}<2^{p} N(r)^{p}$ if $k+j<p$. In view of the above estimates and (7), it is clear that

(25) $|\Phi(z)| \leq K(N(r))^{p}(M(r))^{-1} \exp \left(r^{\varphi(r)}\right)$

where $K$ is a positive constant independent of $r$. Now by $(5), N(r)<\left(c^{-1}\right.$ $\log M(r))^{1 / \alpha}$, and so from (25),

(26) $|\Phi(z)| \leq \Psi(r)(M(r))^{-1 / 2} \exp \left(r^{\varphi(r)}\right)$,

where $\Psi(r)=K\left(c^{-1} \log M(r)\right)^{p / \alpha}(M(r))^{-1 / 2}$. Since $M(r) \rightarrow+\infty$ as $r \rightarrow+\infty$, clearly $\Psi(r) \rightarrow 0$ as $r \rightarrow+\infty$. Thus there exists $R_{5}>R_{4}^{\prime}$ such that, $\Psi(r)<1$ for $r>R_{5}$. Then (23) follows from (26).

We now consider $\Lambda(z)$ (i.e. the left side of (22)).

Case I. $\quad m=0$. Then $\Lambda(z)=f_{p-m, m}(z)$. Since $\Lambda(z)=\Phi(z)$ (by (22)), we have by (12) and (23) that, $\exp \left(-r^{\varphi(r)^{1+\varepsilon}}\right) \leq(M(r))^{-1 / 2} \exp \left(r^{\varphi(r)}\right)$ if $r \in B$ and $r>R_{5}$. Since $\varphi(r)>1, \varphi(r)<\varphi(r)^{1+\varepsilon}$, so we obtain

(27) $M(r) \leq \exp \left(4 r^{\varphi(r)^{1+\varepsilon}}\right)$ if $r \in B$ and $r>R_{5}$. But if $r \in B$ then $r \in A$ so $N(r)>\exp \left(3 r^{\left.\varphi(r)^{1+\varepsilon}\right)}\right.$. Hence by (5), $\log M(r)>c \exp \left(3 \alpha r^{\left.\varphi(r)^{1+\varepsilon}\right)}\right.$, which together with (27) gives

(28) $(c / 4) r^{-\varphi(r)^{1+\varepsilon}} \exp \left(3 \alpha r^{\varphi(r)^{1+\varepsilon}}\right)<1$ if $r \in B$ and $r>R_{5}$. But since $c>0$, $\alpha>0$ and $r^{\varphi(r)^{1+\varepsilon}} \rightarrow+\infty$ as $r \rightarrow+\infty$, it is clear that the left side of (28) tends to $+\infty$ as $r \rightarrow+\infty$. Thus (28) is impossible (since by our assumption (20), there exist $r$-values in $B$ tending to $+\infty$ ). This contradiction proves Lemma $\mathrm{A}$ in the case $m=0$. 
Case II. $m>0$. By (24), if $r \in B$ and $r>R_{5}$ then $|\delta(z)|<1 / 2$, and so at each point of $|z|=r$ at which $|h(z)|=M(r)$, we have by (6) that, $\left|h^{\prime}(z)\right| h(z)|\geq(1-|\delta(z)|) N(r)| r>(2 r)^{-1} N(r)$. But if $r \in B$ then $N(r)>\exp \left(3 r^{\varphi}(r)^{1+\varepsilon}\right)$ (by (19)). Thus if $r \in B$ and $r>R_{5}$, then

(29) $\left|h^{\prime}(z) / h(z)\right| \geq(2 r)^{-1} \exp \left(3 r^{\varphi(r))^{1+\varepsilon}}\right)$,

at each point of $|z|=r$ at which $|h(z)|=M(r)$.

Now we observe that $\Lambda(z)$ can be written in the form,

(30) $\quad \Lambda(z)=f_{p-m, m}(z)\left(h^{\prime}(z) / h(z)\right)^{m}\left(1+\sum_{j=0}^{m-1} \Psi_{j}(z)\right)$, where

(31) $\Psi_{j}(z)=\left(f_{p-j, j}(z) / f_{p-m, m}(z)\right)\left(h^{\prime}(z) / h(z)\right)^{j-m}$, for $j=0,1, \cdots, m-1$. We consider the $\Psi_{j}(z)$ at points on $|z|=r$ at which $|h(z)|=M(r)$, where $r \in B$ and $r>R_{5}$. In view of the estimates given in (7), (12) and (29), we easily see that for $j=0,1, \cdots, m-1$,

(32) $\left|\Psi_{\jmath}(z)\right| \leq(2 r)^{m} \exp \left(-r^{\varphi(r)^{1+\varepsilon}}\right)$.

Since $\varphi(r)>1$, it is clear that the right side of (32) tends to zero as $r \rightarrow+\infty$. Hence there exists $R_{6}>R_{5}$ such that for $r \in B$ and $r>R_{6}$,

(33) $\left|\Psi_{j}(z)\right| \leq 1 /(m+1)$ for $j=0,1, \cdots, m-1$.

Now by (30), $|\Lambda(z)| \geq\left|f_{p-m, m}(z)\right|\left|h^{\prime}(z) / h(z)\right|^{m}\left(1-\sum_{j=0}^{m-1}\left|\Psi_{j}(z)\right|\right)$, and so in view of the estimates given in (12), (29) and (33), we easily see that for $r \in B$ and $r>R_{6}$,

(34) $|\Lambda(z)| \geq(1 /(m+1))(2 r)^{-m} \exp \left((3 m-1) r^{\varphi(r))^{1+\varepsilon}}\right)$

at each point of $|z|=r$ at which $|h(z)|=M(r)$. We may write (34) as,

(35) $|\Lambda(z)| \geq Q(r) \exp \left(r^{\varphi(r)^{1+\varepsilon}}\right)$,

where $Q(r)=(1 /(m+1))(2 r)^{-m} \exp \left((3 m-2) r^{\varphi(r) 1+\varepsilon}\right)$. Now since $m \geq 1$ and $\varphi(r)>1$, clearly $Q(r) \rightarrow+\infty$ as $r \rightarrow+\infty$. Thus there exists $R_{7}>R_{6}$ such that if $r>R_{7}$ then $Q(r)>1$. Hence in view of (35), if $r \in B$ and $r>R_{7}$ then

(36) $|\Lambda(z)|>\exp \left(r^{\varphi(r) 1+\varepsilon}\right)$,

at each point of $|z|=r$ at which $|h(z)|=M(r)$.

Since $\Lambda(z)=\Phi(z) \quad$ by (22)), we have by (23) and (36) that if $r \in B$ and $r>R_{7}$, then $\exp \left(r^{\varphi(r)^{1+\varepsilon}}\right)<(M(r))^{-1 / 2} \exp \left(r^{\varphi(r)}\right)$. Since $\varphi(r)<\varphi(r)^{1+\varepsilon}$, we thus 
obtain,

(37) $M(r)<1$ if $r \in B$ and $r>R_{7}$.

But $M(r) \rightarrow+\infty$ as $r \rightarrow+\infty$ (by (1)), so (37) is impossible (since by our assumption (20) there exist $r$-values in $B$ tending to $+\infty$ ). This contradiction proves Lemma $A$ in Case II and so the proof of Lemma $A$ is complete.

We now prove,

Lemma B. There exists a real number $r_{2}>1$ such that $N(r) \leq \exp \left(3(b r)^{\varphi(b r)^{1+\varepsilon}}\right)$ for all $r \geq r_{2}$ (where $b$ is as in (8)).

Proof. By Lemma A, there exists $r_{1}>1$ such that

(38) $A \cap\left(r_{1},+\infty\right) \subset E \cup F$.

Now by (16), $E \cup F$ is the union of open intervals $\left(U_{s}, U_{s}^{\prime}\right)$ satisfying (17) and (18). In view of (18), $\lim _{s \rightarrow \infty}\left(U_{s}^{\prime} / U_{s}\right)=1$, and by (17), $\lim _{s \rightarrow \infty} U_{s}^{\prime}=+\infty$. Thus since $b>1$ (by (8)), there exists $s_{0}$ such that

(39) $\left(U_{s}^{\prime} / U_{s}\right)<b$ and $U_{s}^{\prime}>r_{1}$ for all $s \geq s_{0}$.

We will show that if $r_{2}$ is taken to be $U_{s_{0}}^{\prime}$, then the conclusion of Lemma $\mathrm{B}$ holds. Let $r \geq r_{2}$.

If $r \notin A$, then by (19), $N(r) \leq \exp \left(3 r^{\left.\varphi(r)^{1+\varepsilon}\right)}\right.$. Since $b>1$ and $\varphi$ is monotone nondecreasing, clearly then $N(r) \leq \exp \left(3(b r)^{\left.\varphi(b r)^{1+\varepsilon}\right)}\right.$.

If $r \in A$, then by (38), $r \in E \cup F$. Hence for some $t, r \in\left(U_{t}, U_{t}^{\prime}\right)$. Since $U_{s_{0}}^{\prime}=r_{2} \leq r<U_{t}^{\prime}$, we have $t>s_{0}$ (by (17)), and so (39) holds for $s=t$. Now in view of (17), the endpoint $U_{t}^{\prime}$ is clearly not in $E \cup F$. Hence by

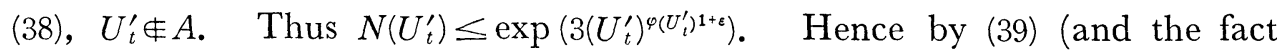
that $\varphi$ is monotone nondecreasing), we have $N\left(U_{t}^{\prime}\right) \leq \exp \left(3\left(b U_{t}\right)^{\left.\varphi\left(b U_{t}\right)^{1+\varepsilon}\right)}\right.$. Since

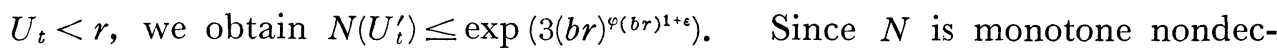

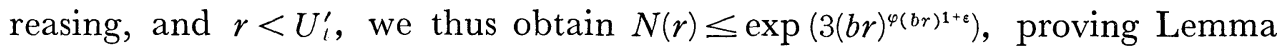
B completely.

Now for each $r \geq 0$, let $q(r)$ be the maximum term [6; p. 193] of $h$ (i.e. if $\sum_{n=0}^{\infty} c_{n} z^{n}$ is the power series expansion of $h$, then $\left.q(r)=\max _{n \geq 0}\left|c_{n}\right| r^{n}\right)$. It is proved in $[6 ; \mathrm{p} .195]$ that for any $r_{0}>0$,

(40) $\log q(r)=\log q\left(r_{0}\right)+\int_{r_{0}}^{r}(N(x) / x) d x$. 
Applying (40) with $r_{0}$ equal to $r_{2}$ (where $r_{2}$ is as in Lemma B), and observing that for $r_{2} \leq x \leq r, N(x) / x \leq N(x) \leq N(r)$ (since $r_{2}>1$ and $N$ is monotone nondecreasing), we obtain,

(41) $\log q(r) \leq \log q\left(r_{2}\right)+\left(r-r_{2}\right) N(r)$ for all $r \geq r_{2}$. But by Lemma B, $N(r) \leq \exp \left(3(b r)^{\varphi(b r)^{1+\varepsilon}}\right)$ for $r \geq r_{2}$, and since $\log q\left(r_{2}\right)$ is a constant, it follows easily from (41) that there exists $r_{3}>r_{2}$ such that

(42) $\log q(r) \leq \exp \left(4(b r)^{\left.\varphi(b r)^{1+\varepsilon}\right)}\right.$ for all $r \geq r_{3}$.

Now it is proved in $[8 ;$ p. 106] that the following relation holds between $M(r)$ and $q(r)$ : For any $\varepsilon_{1}>0$, there exists $r_{4}>r_{3}$ such that if we remove from $\left(r_{4},+\infty\right)$ an infinite sequence of exceptional finite open intervals $\left(V_{s}, V_{s}^{\prime}\right)$ for which

(43) $\sum_{s=1}^{\infty}\left(\log V_{s}^{\prime}-\log V_{s}\right)$ converges, and for which we may assume that

(44) $V_{s}^{\prime}<V_{s+1}$ for all $s$ and $\lim _{s \rightarrow \infty} V_{s}^{\prime}=+\infty$, then for all $r$ in $\left(r_{4},+\infty\right)-G$, where $G=\bigcup_{s=1}^{\infty}\left(V_{s}, V_{s}^{\prime}\right)$, we have,

(45) $M(r)<q(r)(\log q(r))^{{ }_{1}+(1 / 2)}$.

We apply the above with $\varepsilon_{1}=1 / 2$, and using (42), we obtain for $r>r_{4}$ and $r \notin G$,

(46) $\log M(r)<\exp \left(4(b r)^{\varphi(b r)^{1+\varepsilon}}\right)+4(b r)^{\varphi(b r)^{1+\varepsilon}}$.

Hence clearly there exists $r_{5}>r_{4}$ such that

(47) $\log M(r)<\exp \left(5(b r)^{\left.\varphi(b r)^{1+\varepsilon}\right)}\right.$ for $r>r_{5}$ and $r \notin G$.

We now prove,

Lemma C. There exists $r_{6}>1$ such that

(48) $\log M(r)<\exp \left(5(a r)^{\varphi(a r)^{1+\varepsilon}}\right)$,

for all $r \geq r_{6}$ (where $a$ is as in (8)).

Proof. In view of (43), clearly $\left.\lim _{s \rightarrow \infty} V_{s}^{\prime} / V_{s}\right)=1$. Since $b>1$ (by (8)) and $\lim _{s \rightarrow \infty} V_{s}^{\prime}=+\infty$ (by (44)), there exists $t_{0}$ such that

(49) $V_{s}^{\prime} / V_{s}<b$ and $V_{s}^{\prime}>r_{5}$ if $s \geq t_{0}$. 
Set $r_{6}=V_{t_{0}}^{\prime}$ and let $r \geq r_{6}$. If $r \notin G$ then (48) follows from (47) in view of the fact that $\varphi$ is monotone non-decreasing and $b<b^{2}=a$ (by (8)).

If $r \in G$, then $r \in\left(V_{t}, V_{t}^{\prime}\right)$ for some $t$. In view of (44), $t>t_{0}$ so (49) holds for $s=t$. Clearly the endpoint $V_{t}^{\prime}$ is not in $G$ so (47) holds for the point $V_{t}^{\prime}$. Since $\varphi$ is monotone nondecreasing and $V_{t}^{\prime}<b V_{t}$ (by (49)), we then obtain $\log M\left(V_{t}^{\prime}\right)<\exp \left(5\left(b^{2} V_{t}\right)^{\varphi\left(b^{2} V_{t}\right)^{1+\varepsilon}}\right)$. Since $V_{t}<r<V_{t}^{\prime}$ and $\log M$ is increasing (and noting that $b^{2}=a$ ), it is now clear that (48) holds for $r$, proving Lemma $\mathrm{C}$.

We now conclude the proof of our theorem. By hypothesis, $\varphi(r)>1$ for $r \geq R_{0}$. Let $K_{0}=\varphi\left(R_{0}\right)^{\varepsilon}-1$. Then $K_{0}>0$, so there exists $r_{0}>\max$ $\left\{R_{0}, r_{6}\right\}$ such that $(\log a / \log r)<K_{0} / 2$ and $(\log 5 / \log r)<K_{0} / 2$ for all $r>r_{0}$. Since $\varphi$ is monotone nondecreasing and $\varphi(a r)>1$, we obtain $\varphi(a r)^{1+\varepsilon}\left(\varphi(a r)^{\varepsilon}-\right.$ $((\log a / \log r)+1))>K_{0} / 2>\log 5 / \log r$ for $r>r_{0}$. It now easily follows from (48) that $\log M(r)<\exp \left(r^{\varphi(a r)^{1+2 \varepsilon}}\right)$ for $r>r_{0}$, and since $1+2 \varepsilon<a$ (by (8)), we obtain finally $M(r)<\exp \left(\exp r^{\varphi(a r)^{a}}\right)$ for $r>r_{0}$, which concludes the proof of the theorem.

5. Remark: In this remark, we consider the application of our main result to meromorphic solutions $H(z)$ of $\Omega\left(z, y, y^{\prime}\right)=\sum f_{k j}(z) y^{k}\left(y^{\prime}\right)^{j}=0$ (i.e. meromorphic functions $H(z)$ such that $\Omega\left(z, H(z), H^{\prime}(z)\right)=0$ at each point $z$ at which $H$ is analytic). We observe first that if $H(z)$ is a meromorphic solution of $\Omega\left(z, y, y^{\prime}\right)=0$, say $H(z)=h(z) / g(z)$, where $h$ and $g$ are entire functions, then $h$ and $g$ satisfy the relation,

(50) $\sum f_{k j}(z)(g(z))^{n-(K+2 \jmath)}(h(z))^{k}\left(g(z) h^{\prime}(z)-h(z) g^{\prime}(z)\right)^{j} \equiv 0$,

where $n=\max \left\{k+2 j: f_{k j} \not \equiv 0\right\}$. Thus the entire function $h(z)$ satisfies the algebraic differential equation (50) whose coefficients belong to the subring of the ring of entire functions generated by $g, g^{\prime}$ and the $f_{k j}$. Similarly, $g$ satisfies the equation (50) whose coefficients belong to the subring generated by $h, h^{\prime}$ and the $f_{k j}$. These observations, together with our main result, permit us to obtain information on the growth of $h$ (respectively, $g$ ), if we have growth information on $g$ (respectively, $h$ ) and the $f_{k j}$. Since $H=h / g$, we will then have growth information on $H$ (using, of course, the Nevanlinna characteristic to measure the growth).

As a simple example of this observation, suppose each coefficient $f_{k j}$ is of finite order, and suppose $H=h / g$ is a meromorphic solution of $\Omega\left(z, y, y^{\prime}\right)=0$, where $g$ is of finite order. Then (50) shows that $h$ is an entire solution of 
an equation whose coefficients are of finite order. By our theorem (taking $\varphi(r)$ in our theorem to be a sufficiently large constant), we have that there exist constants $\sigma>1$ and $r_{0}>1$ such that $M(r ; h) \leq \exp \left(\exp r^{\sigma}\right)$ for all $r>r_{0}$. Since $g$ was assumed to be of finite order, it follows easily (using [4; pp. $15,24])$, that for some constant $d>0$, the Nevanlinna characteristic $T(r, H)$ of $H(z)$ satisfies the inequality, $T(r, H) \leq \exp \left(r^{d}\right)$ for all sufficiently large $r$. (By a symmetric argument, clearly the same conclusion holds if $h$, instead of $g$, is assumed to be of finite order).

\section{BiBLIOGRAPHY}

[1] S. Bank, On solutions of algebraic differential equations whose coefficients are entire functions of finite order, Ann. Mat. Pura Appl., 83 (1969), 175-184.

[2 ] O. Blumenthal, Principes de la théorie des fonctions entières d'ordre infini, GauthierVillars, Paris, 1910.

[ 3 ] E. Hille, Analytic Function Theory--Volume II, Ginn and Company, Boston, 1962.

[4] R. Nevanlinna, Le théorème de Picard-Borel et la théorie des fonctions méromorphes, Gauthiers-Villars, Paris, 1929.

[5] S. Saks and A. Zygmund, Analytic Functions, Warsaw, 1952.

[6] G. Valiron, Fonctions Analytiques, Presses Universitaires de France, Paris, 1954.

[ 7 ] G. Valiron, Sur les fonctions entieres vérifiant une classe d'équations différentielles, Bull. Soc. Math. France, 51 (1923), 33-45.

[ 8 ] G. Valiron, Lectures on the general theory of integral functions, Edouard Privat, Toulouse, 1923.

[ 9 ] G. Valiron, Les théorèmes généraux de M. Borel dans la théorie des fonctions entières, Ann. Ecole Norm., t. 37 (1920), 219-253.

[10] A. Wiman, Ueber den Zusammenhang zwischen dem Maximalbetrage einer analytischen Funktion und dem grössten Gliede der zugehörigen Taylorschen Reihe, Acta Math., 37 (1914), 305-326.

[11] A. Wiman, Ueber den Zusammenhang zwischen dem Maximalbetrage einer analytischen Funktion und dem grössten Betrage bei gegebenem Argumente der Funktion, Acta Math., 41 (1916), 1-28.

University of Illinois 